\title{
THE MUON SOURCE WITH SMALL PHASE-SPACE PRODUCED FROM THE COMPRESSED TARGET FOR MUON-MUON COLLIDER
}

\author{
Hiroshi Takahashi and Jun Zhang \\ Brookhaven National Laboratory, Upton, NY 11973
}

\begin{abstract}
For the muon-muon collider, we proposed using a target compressed by high intensity laser or heavy-ions irradiation, to collect the pions produced in very small phase-space and then to cool them by another high intensity laser in the PAC 97. Although the pions are produced initially with small phase space, they will be spread transversely in a short time because of their large transverse momentum; consequently a broad highintensity laser irradiation is needed to collect and accelerate them, and thus the process is not economical. To keep the transverse dimension small, a high solenoidal magnetic field is needed. This field can be created using circularly polarized laser irradiation to the target's surface. Amagnetic field of mega-tesla order of magnetic field can be created by the laser intensity of $10^{15} \mathrm{watt} / \mathrm{cm}^{2}$, using approach developed Eliser; the Lahmer radius of the produced pions becomes less than $0.01 \mathrm{~mm}$, and they can be accelerated by another laser irradiation while in this confined state. Since the target is compressed at not a high temperature, the energy required for this compression is much smaller than the inertial confinement fussion-device, and the higher density reduces the target's mass. This approach is more effective than the one used in the RF field for producing cold muons. This approach also can be applied for anti-baryon production.
\end{abstract}

\section{INTRODUCTION}

To achieve a luminosity of $10^{35} \mathrm{~cm}^{-2} \mathrm{~s}^{-1}$ for a $2+2 \mathrm{Tev}$ muon-muon collider machine as well as a luminosity of $10{ }^{33} \mathrm{~cm}^{-2} \mathrm{~s}^{-1}$ for a $250+250 \mathrm{GeV}$ collider [1], it is necessary to produce and collect a large number of muons. The basic method presently used starts with a proton beam impinging on a thick target (one to two interaction lengths), followed by a long solenoid which results mainly from the decay of pions. Since a substantially large amount of the energy is deposited to the small target, heat removal from it is one of most important problems in designing the target to efficiently collect pions. The energy needed, and the cost of collecting the secondary beam using the high solenoid magnetic field and RF cavity is very high. To reduce the energy cost of producing and collecting the muons which result from the decay of the pions, the approach of using a highly compressed target[2], which is practiced in inertial fusion, is promising.
A $10^{18}$ watt $/ \mathrm{cm}^{2}$ laser beam-power can create an electric field of $10^{9} \mathrm{~V} / \mathrm{cm}$ by using inverse cherenkov radiation[3]. An extensive study is underway on the acceleration of electrons using a laser, such as a plasma acceleration, two beat wave-beams acceleration using a strong laser intensity $[4,5]$. By modulating a high intensity laser, we might control the secondary particles produced by a high-power accelerator in a similar way as laser acceleration.

By using the RF cavity, the secondary particles produced are confined with a high magnetic field of a few tens Tesla, and the phase rotation cavity is located far from the target area; the secondary beam is elongated during its travel from the point of generation to this cavity, and long cavity is required which has low frequency RF.

When secondary particles are created from a small sized target of high density, the particles are small in size and can be controlled directly just after their creation by a strong laser field. The cavity is not needed.

\section{COMPRESSED TARGET}

In an inertial-confinements fusion experiment, a high density of 600 times that of the density of liquid hydrogen was achieved by compressing the target with $15 \mathrm{KJ}$ pulsed-laser irradiation [6,7]. When a target is compressed to 1000 times its original density, and highenergy protons impinge on it, then pions are produced at a 1000 times higher density.

When the size if the original uncompressed target is $1 \mathrm{~cm} \cdot 1 \mathrm{~cm} \cdot 10 \mathrm{~cm}$, by compressing the target 1000 times, the size of the compressed target in to which the protons are injected becomes $0.001 \mathrm{~cm} \cdot 0.001 \mathrm{~cm} \cdot 0.01 \mathrm{~cm}=10^{-8} \mathrm{cc}$. Further, the total number of $\mathrm{Pb}$ atoms in the compressed target is $\left(0.3 \cdot 10^{23} \cdot 10^{-8}=0.3 \cdot 10^{15}\right) \cdot 10^{3}=0.3 \cdot 10^{18}$; if we assume that $\mathrm{Pb}$ atoms in the compressed state have 100 ev potential energy, then the total potential energy of the compressed target is $3 \cdot 10^{19} \mathrm{eV}\left(/ 6 \cdot 10^{18} \mathrm{eV} / \mathrm{J}\right)=5$ Joules. If it is further assumed that 1000 times the driving energy is required to achieve this compressed state, then the total driving energy to compress this target is $5 \mathrm{~K}$ joule.

According to hydrodynamic simulations [8] for laserdriven implosions, a DT solid layer of $0.250 \mathrm{~mm}$ thickness for $2.250 \mathrm{~mm}<\mathrm{r}<2.5 \mathrm{~mm}$ contained in a polyethylene shell of $0.045 \mathrm{~mm}$ thick can be compressed 
by a $600 \mathrm{~g} / \mathrm{cc}$, with a radius from 0.06 to $0.1 \mathrm{~mm}$ after a 35.5 nsec layer irradiation. This high density can be maintained for about $2 \mathrm{n} \mathrm{sec}$.

The passing time of $30 \mathrm{GeV}$ protons with a beam length of $100 \mathrm{~cm}$ is shortened to $100 \mathrm{~cm} / 3 \cdot 10^{10}(\mathrm{~cm} / \mathrm{sec}) / 30=0.11$ $\mathrm{n} \mathrm{sec}$; this time is far shorter than the above compressing time of $2 \mathrm{n} \mathrm{sec}$.

\section{PION PRODUCTIONS}

To produce the pions, the heavy nuclei, such as $\mathrm{Pb}$, are more effective than light nuclei, but to compress these high- $\mathrm{Z}$ materials a higher energy is required. When a light nuclei target, such as Be, is compressed to 1000 times its normal density, the radiation length is shortened by factor 1000 , but the probability of proton collisions is reduced about a 10 fold smaller factor for Be target with the same size radius; thus $90 \%$ of the incident protons pass through without colliding with the target. To increase the probability of collision, the cylindrical geometry target should be compressed by employing a Z-pinch-type inertial fusion method, instead of spherical geometrical compression.

To use the uncollided protons, tandem targets which are equivalent to a cylindrical target geometry can be used, or the protons can be recirculated adding accelerating energy. The latter option needs a more complicated engineering design, so it is more practical to use a cylindrical target with longer axial length. The production rate of pions from the light nuclei is smaller than that from heavy nuclei because of the smaller numbers of intra-nuclear cascade processes that occur in the nuclear collisions. Although the light-nuclei target reduces the production probability per one injecting proton compared with the heavy nuclei target, the use of the light nuclei target with long axial length might be more practical than using a heavy nuclei target. These alternatives should be studied by the nuclear-cascade code and by simulating compression processes, not only laser or heavy-ion compression or z-pinch machines.

The compressed target is impinged upon by the highenergy protons with an energy above $30 \mathrm{GeV}$, and focused into radius of $0.06 \mathrm{~mm}$. When the primary proton number in one pulse is $10^{13}$ then the density of protons becomes $10^{14} \mathrm{P} / \mathrm{cc}$. By focusing the beam, the problem of charge space can be resolved.

By employing a compressed target and a proton beam, secondary particles are produced with a high luminosity. However, the secondary particles have a high transverse momentum; thus, they will quickly be spread, and so large RF powers are required to focus and control the secondary beam, similar to the case of using an uncompressed target.

\section{SOLENOIDAL MAGNETIC FIELD CREATED BY CIRCULAR POLARIZED BEAM}

To confine the secondary particles in a small cylindrical space, a solenoidal magnetic field is needed. By imposing a high-intensity solenoidal $\mathrm{Bz}$ magnetic field to the secondary beam just after its production, its transverse dimension can be kept small. Elizer [9] suggested that a mega-gauss magnetic solenoidal field can be generated using a circularly polarized laser light (CPLL).

The generation of the Bz field by CPLL is formulated as

$$
\begin{aligned}
B(\text { Gauss })= & 2 \cdot 10^{4} \cdot\left[\mathrm{I}_{\mathrm{M}} \cdot 10^{14} \mathrm{~W} \cdot \mathrm{cm}^{-2}\right] \\
& \cdot[\lambda / \mu \mathrm{m}]^{3} \cdot\left[\mathrm{n} / 10^{21} \mathrm{~cm}^{-3}\right],
\end{aligned}
$$

where $\mathrm{B}$ is the magnetic field, $\lambda$ is the laser wave-length, $\mathrm{I}$ is the laser intensity, and $\mathrm{n}$ is the plasma density.

Using a $\lambda=10$. micron wave-length laser with a high intensity of $10^{16} \mathrm{~W} / \mathrm{cm}^{2}$ to $\mathrm{n}=10^{20}$ plasma density, a magnetic field of $200 \mathrm{MG}$ can be produced. The $\lambda$ radius of pions and muons with $100 \mathrm{MeV}$ transverse kinetic energy in this high magnetic field is $0.003 \mathrm{~cm}$. By irradiating the target just after their production with this high-intensity laser of $10^{16} \mathrm{~W} / \mathrm{cm}^{2}$ in a radius of $0.006 \mathrm{~cm}$, the pions and muons can be confined in a cylinder with a $0.006 \mathrm{~cm}$ radius. The total laser power to create this field is $10^{14} \mathrm{Watt}$, and if we confine the beam for $30 \mathrm{n} \mathrm{sec}$, we need to have $30 \mathrm{KJ}$ of laser energy.

As shown in Eq.1, the laser power required to create a high magnetic field is proportional to cubic power of the wave-length of laser, and inversely proportional to plasma's, electron-density. The use of a longer wavelength laser is beneficial, but the plasma's electron density affects the penetration of the laser into the target plasma frequency also becomes high and then a laser with a frequency less than this plasma frequency not can penetrate; hence, a circularly polarized laser not can create a high current and a high solenoidal field. A high frequency laser can penetrate more deeply into plasma, so that a high $\mathrm{Bz}$ field can be created; however the laser power then required to generate a high $\mathrm{Bz}$ field is proportional to the cube of the laser's frequency, and so the laser power needed becomes prohibitively high for practical purposes.

In this paper, we did not optimize our study for designing target compression nor for the use of the high intensity laser for controlling the secondary particles; we simply analyzed the orders of magnitude of the required laser energy. This value is much smaller than the conventional $\mathrm{RF}$ field. In further analyses, we need to simulate compression ( laser, heavy ions, Z-pinch type), and laserplasma interactions, as well as making nuclear-cascade calculations. 
To compress the target material initially requires a large amount of energy for driving devices, such as a laser, light- and heavy-ions drivers, the substantial expense of energy required for controlling the secondary particles can be saved in this approach. The reduction of the emittance confers a tremendously high cost-benefit advantage.

This approach of using the laser is especially useful for collecting the anti-protons which cannot use ionization cooling because of its annihilation of anti-protons.

The technology of accelerating a charged beam by laser is still in its infancy, but this technology also should be applied to target technology.

To compress the target material, we can employ a high-z pinch device instead of irradiation by laser, light- and heavy-ions. This suggested approach to compression is much simpler than the others.

\section{ACKNOWLEDGMENT}

The authors would like to express their thanks to Drs. R.Palmer, A.N.Skrinsky, F.E.Mills J.Gallardo, S. Eliezer, C.Yamanaka, H.Takabe, This work was performed under the auspices of the U.S. Department of Energy under Contract No. DE-AC02-76CH00016.

\section{REFERENCES}

1. R.Palmar et al. "Muon Collider Design " BNL62949(1996), "Muon Collider" 9th advanced ICFA, Beam Dynamics and Technology Issue for $\mathrm{Mu}-\mathrm{Mu}$ collider, Beam Dynamics Workshop. Oct. 1995, AIP Conf. 372, p.3. Montauk, NY, 1995 Edited by J. Gallardo. 2.H. Takahashi, Y.An, X.Chen, M. Nomura “ Optimization of the Target for Muon Colliders" Proc. of the 1997 particle Accelerator conference (PAC-97), p.402 Vancouver ,B.C., Canada 12-16, May 1997.

3. A. Fisher, J.Gallardo, J. Sandweiss, A. Van Steenbergen, " Inverse Free- Electron Laser Accelerator P.299. AIP Conference Proceeding 279 Advanced accelerator concepts Port Jefferson, NY 1992. Editor J. S. Wurtelle.

4. T. Tajima and J.M.Dawson Phys. Rev. Lett. 43, 267 (1979)

5. T. Katsouleas " Summary of the working group on Plasma Accelerator' P371, C.M. Tang "Report of the working group on Far Field Accelerators, p. 259,

6. C. Yamanaka " Advances in inertial Confinement Fusion" OR3.1,ICENES'89, Karlsruhe, 1989.

7. H.Azechi et al 1991, Laser and Particle beams 9, 193, 1991.

8. H. Takabe " Hydrodynamic Simulation Code for Laser Driven Implosion" Private communication, oct, 97.

9. E.Kolka, S.Eliezer, Y.Paiss, "Miniature Magnetic Bottle confined by cicularly polarized laser light “

Laser and Particle Beams (1995) 13, 83-93. 